\title{
Observations sur trois cas de Cysticercose musculaire généralisée
}

\author{
par Ión GHERMAN, Mircea DEBAU, Sorin SCHIAU et Marianne DEBAU
}

Les cas de cysticercose chez l'homme apparaissent, on le sait, après l'ingestion d'embryophores de Taenia solium ${ }^{\left({ }^{*}\right)}$ avec l'eau ou les aliments contaminés (hétéroinfestation). L'auto-infestation chez les personnes infestées est aussi possible par deux mécanismes :

1) régurgitation d'anneaux du parasite de l'intestin dans l'estomac (auto-infestation endogène) ;

2) ingestion d'embryophores éliminés à l'extérieur et arrivant de nouveau dans l’organisme par les mains contaminées (auto-infestation exogène) (v. fig. 1) (6).

Ainsi par exemple, un cas de cysticercose relativement récent, présenté par Helimsky K. A. (1962) (8), est consécutif à une auto-infestation chez une malade de 19 ans, parasitée par un T. solium.

La cysticercose musculaire de l'homme, déterminée par la localisation dans les muscles des formes larvaires (Cysticercus cellulosae) de $T$. solium, est une parasitose très rare. Cela est dû au fait que les cas de taeniasis sont en général assez rares, et que parmi eux, le nombre de porteurs de $T$. solium, engendrant habituellement la cysticercose, ne constitue, au moins chez nous, d'après V. Nitzulescu et coll. (1955) (10) et I. Gherman et E. Zaharia (1960) (7), qu'un pourcentage d'environ $10 \%$. D'autre part, la rareté des cas de cysticercose musculaire s'explique encore par le fait que les cysticerques se localisent plus fréquemment dans les yeux ou le système nerveux que dans les tissus sous-cutanés ou dans les muscles. Cela résulte aussi de la statistique plus ancienne de J. Vosgien (cité par E. Brumpt) (1959) (2), d'après laquelle les cysticerques se fixent dans l'œil et ses annexes dans $46 \%$ des cas, dans le système nerveux dans $40,99 \%$, dans la peau et le tissu cellulaire dans $6,32 \%$ et seulement dans $3,47 \%$ des cas dans les muscles. Ce faible pourcentage des localisations musculaires est confirmé par E.-R. Brygoo et A. Dodin (1963) -(3), qui, sur 59 cas de cysticercose dépistés à Madagascar, ne signalent que sept cas de cysticercose musculaire $(5,18 \%)$.

(*) Les embryophores de Taenia saginata interviennent tout à fait execeptionnellement dans l'étiologie de la cysticercose chez l'homme. 
Dans la littérature médicale de notre pays, ces cas de cysticercose sont tout à fait exceptionnels. En ce qui concerne les observations récentes, en dehors des cas mentionnés par V. Nitzulescu (1964) (11), un cas a été signalé par G. Berlinschi et coll. (1963) (1).

Au cours de ces dernières années, deux d'entre nous (radiologues) ont essayé de dépister parmi nos malades des cas éventue's de cysticercose.

C'est ainsi que trois cas de cysticercose musculaire généralisée, dont les images radiologiques sur les différents segments sont présentées dans les figures 2,3 et 4 , ont été identifiés.

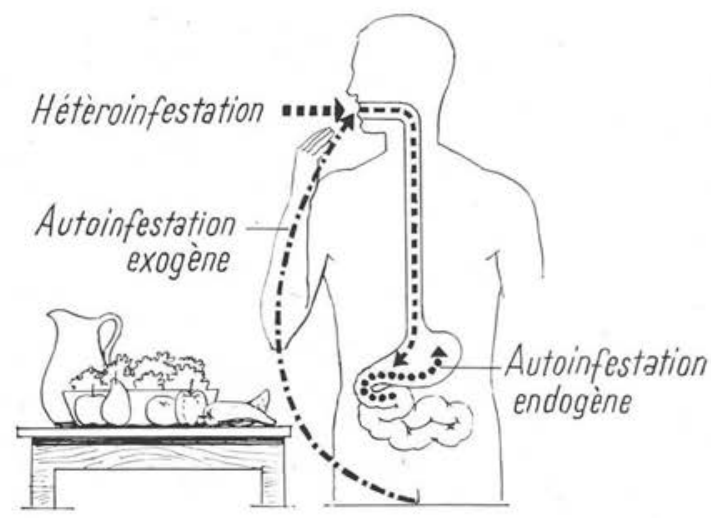

Fig. 1. - Les différents modes d'infestation dans la cysticercose humaine

Les cysticerques ont une opacité calcaire ; de forme ovalaire, ils mesurent $2-4 \mathrm{~mm}$ Les cysticerques ont une opacité ca!caire ; de forme ovalaire, ils mesurent 2-4 mm. de large et jusqu'à $10 \mathrm{~mm}$. et plus de long, bien qu'ils soient différemment décrits par tation (5), ce qui prouve l'ancienneté de l'infestation de nos malades. Dans un cas au moins, le début de la maladie, plus nettement marqué par l'apparition d'une crise comitiale (probablement due à une localisation cérébrale des cysticerques), s'est produit six ans auparavant. Les cysticerques, quelquefois très nombreux, se répartissent dans des régions symétriques, comme l'a remarqué Dang Vu Hy et coll. (1960) (4), dans la cysticercose cutanée.

Du point de vue clinique, un cas seulement ( $n^{\circ} 1$ du tableau ci-joint) a eu une symptomatologie plus riche (céphalalgie, asthénie et troubles neuropsychiques de type comitial), déterminée, comme nous l’avons mentionné, par une cysticercose cérébrale associée. Le symptôme majeur et constant chez tous les malades, consiste en des douleurs musculaires plus ou moins accentuées. L'éosinophilie était insignifiante. 

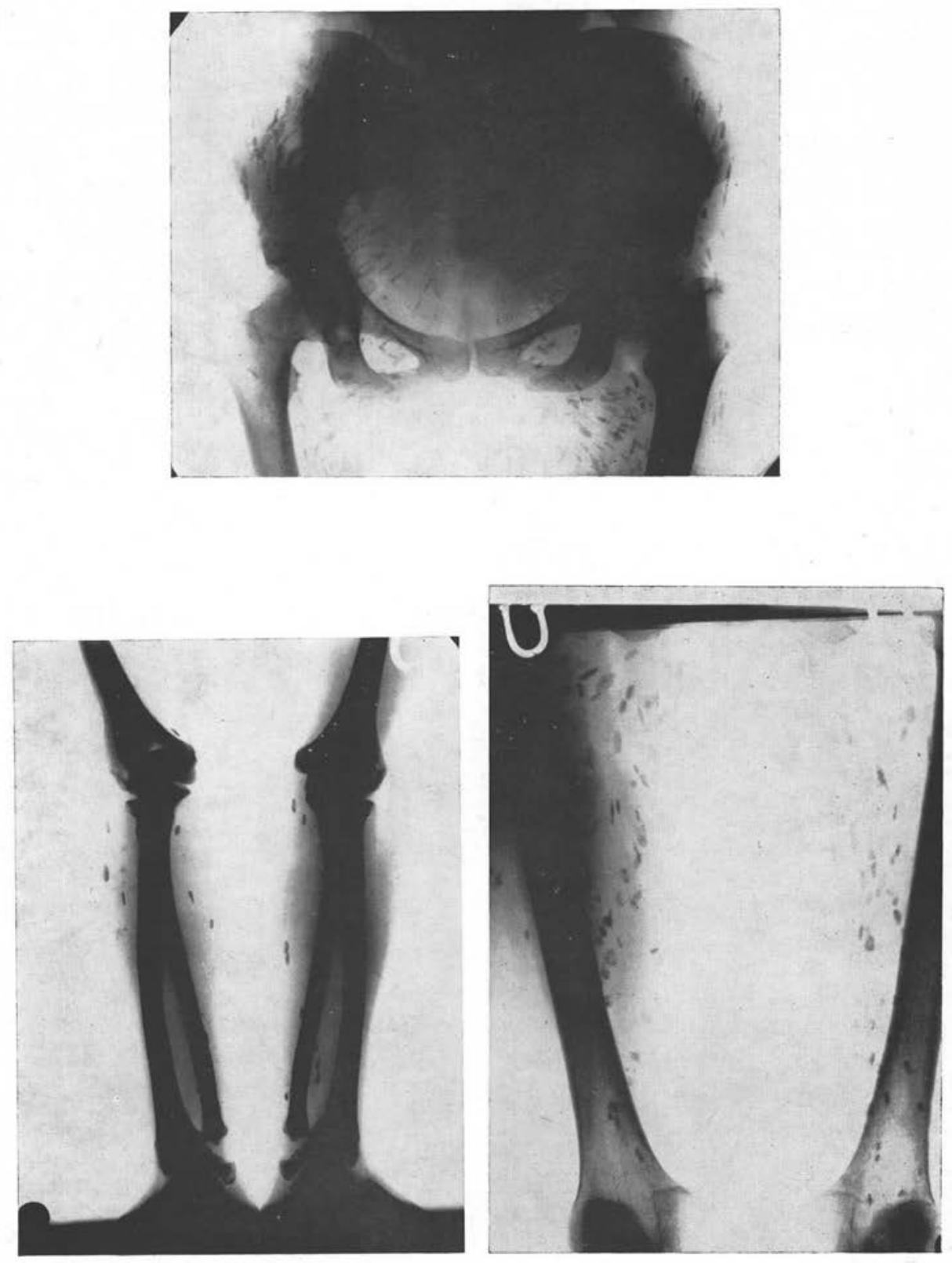

Fig. 2, 3, 4. - Images radiologiques de trois cas de cysticercose musculaire 


\begin{tabular}{|c|c|c|c|c|c|c|}
\hline $\begin{array}{l}\mathrm{N}^{\circ} \\
\text { des } \\
\text { cas }\end{array}$ & Malades & Sexe & Age & Anamnèse & $\begin{array}{l}\text { Eosino- } \\
\text { philie }\end{array}$ & $\begin{array}{c}\text { Etat } \\
\text { présent }\end{array}$ \\
\hline 1. & M. .C. & F & 36 ans & $\begin{array}{l}\text { Crises comitiales, cé- } \\
\text { phalalgie, asthénie, } \\
\text { douleurs musculaires. }\end{array}$ & $8 \%$ & $\begin{array}{l}\text { Médio- } \\
\text { cre }\end{array}$ \\
\hline 2. & E. T. & $F$ & 31 ans & $\begin{array}{l}\text { Douleurs lombaires } \\
\text { et douleurs musculai- } \\
\text { res généralisées. }\end{array}$ & $5 \%$ & Bon \\
\hline 3. & O. B. & M & 51 ans & Douleurs musculaires & $7 \%$ & Bon \\
\hline
\end{tabular}

Des images radiologiques typiques, confrontées avec le tableau clinique, nous ont permis de faire le diagnostic de cysticercose sans que soient nécessaires la biopsie ou un autre examen de contrôle.

Enfin, il faut aussi mentionner que la maladie s'est produite probablement par hétéro-infestation, car aucun sujet n'avait préalablement souffert de taeniasis.

Ces observations, effectuées au cours de l'examen médical d'un grand nombre de personnes, nous permettent d'affirmer que la cysticercose musculaire est un phénomène extrêmement rare. Cependant, un examen radiologique attentivement fait sur les différents segments du corps peut parfois conduire au dépistage de cas de cysticercose musculaire, qui autrement, eussent échappé au diagnostic. C'est pourquoi, bien que la cysticercose musculaire ne soit pas, jusqu'à présent, justiciable d'un traitement efficace (d'ailleurs quelquefois inutile), l'interne, le rhumatologue, le neurologue et surtout le radiologue doivent toujours penser à son existence éventuelle.

\section{Résumé}

Les auteurs décrivent trois cas de cysticercose musculaire généralisée (fig. 2, 3 et 4), dépistés au cours d'examens radiologiques effectués pendant plusieurs années.

A l'exception d'un seul cas où le sujet a présenté, six ans auparavant, des troubles neuropsychiques de type comitial (probablement consécutifs à une localisation initiale cérébrale des cysticerques), les trois cas précités ont eu comme symptôme majeur des douleurs musculaires plus ou moins accentuées. Les malades n'ont pas présenté de taeniasis antérieurement, et l'éosinophilie sanguine est restée à un taux insignifiant.

En conclusion, les auteurs estiment que des examens radiologiques attentivement faits sur les différents segments du corps, peuvent permettre le dépistage de cas de cysticercose musculaire qui eussent autrement échappé au diagnostic du praticien. 


\section{Bibliographie}

1. Berlinschi (G.), Iacomi (N.), Munteanu (R.), Kluger (A.), 1963. - Rev. Med. Chirurgicală, Iași, nr. 3, p. 157.

2. Brumpt (E.), 1959. - Précis de Parasitologie, Paris, 1949. Masson et $C^{1 e}$, édit.

3. Brygoo (R. E.), Dodin (A.), 1963, Arch. Inst. Pasteur, Madagascar, XXXII, 1, p. 37.

4. Dang-Vu-Hy, Huguk-Kham, Le Kinh-Due, Nguyem-Van-Dong, Vi Phac, 1960. - Dermato-Venerologia, nr. 6, p. 481.

5. Dixon (H.), Lipscomb (E.), 1961. - Cysticercosis. London.

6. Gherman (I.), 1958. - Elemente de epidemiologie a bolilor parazitare. Ed. Medicală București.

7. Gherman (I.), Zaharia (Eugenia), 1960. - Studii și cercetări medicale, nr. 1, București, p. 303.

8. Helimscky (A. M.), 1962. - Med. parazit. i parazit. bol. nr. 5. p. 610-611.

9. Moldenhauer (W.), Ziegler (K), 1960. - Ztsch. f. Tropenmed. u, Parasit. Stuttgart, v. 11 , nr. 4.

10. Nitzulescu (V.), Simionescu (V.), Lucian (D.), Popescu (I.), Pescaru (E.), 1955. Pediatria, nr. 4, p. 53-56.

11. Nitzulescu (V.), 1964. - Comunicare persoanlă.

12. SchinZ (H. R. ),1952. - Lehrbuch der Roengendiagnostik, G. Thieme, Stuttgart.

( $V^{\text {e } C l i n i q u e ~ M e ́ d i c a l e ~-~ D i r e c t e u r ~: ~ P r o f . ~ T . ~ S P i ̂ r c h e z, ~ B u c a r e s t, ~ B d ~ 1-M a i, ~ 37-39, ~ R o u m a n i e) ~}$ 